\title{
BP Piscium: its flaring disc imaged with SPHERE/ZIMPOL*
}

\author{
J. de Boer, ${ }^{1,2} \dagger$ J. H. Girard, ${ }^{2}$ H. Canovas, ${ }^{3}$ M. Min,,${ }^{4,5}$ M. Sitko, ${ }^{6,7}$ C. Ginski, ${ }^{1}$ \\ S. V. Jeffers, ${ }^{8}$ D. Mawet,${ }^{9}$ J. Milli, ${ }^{2}$ M. Rodenhuis, ${ }^{1}$ F. Snik ${ }^{1}$ and C. U. Keller ${ }^{1}$ \\ ${ }^{1}$ Leiden Observatory, Universiteit Leiden, PO Box 9513, NL-2300 RA Leiden, the Netherlands. \\ ${ }^{2}$ European Southern Observatory, Casilla 19001, Santiago, Chile. \\ ${ }^{3}$ Departamento de Física Teórica, Universidad Autónoma de Madrid, Cantoblanco 28049, Madrid, Spain. \\ ${ }^{4}$ SRON Netherlands Institute for Space Research, Sorbonnelaan 2, NL-3584 CA Utrecht, the Netherlands. \\ ${ }^{5}$ Astronomical Institute Anton Pannekoek, University of Amsterdam, Science Park 904, NL-1098 XH, Amsterdam, the Netherlands. \\ ${ }^{6}$ Department of Physics, University of Cincinnati, Cincinnati, OH 45221-0011, USA \\ ${ }^{7}$ Center for Extrasolar Planetary Studies, Space Science Institute, Boulder, CO 80301, USA \\ ${ }^{8}$ Institut fuer Astrophysik, Georg-August-Universitaet Goettingen, Friedrich-Hund-Platz 1, D-37077 Goettingen, Germany \\ ${ }^{9}$ Department of Astronomy, California Institute of Technology, 1200 E. California Blvd., Pasadena, CA 91125, USA
}

Accepted 2016 October 20. Received 2016 October 20; in original form 2016 May 15

\begin{abstract}
Whether BP Piscium (BP Psc) is either a pre-main sequence $\mathrm{T}$ Tauri star at $d \approx 80 \mathrm{pc}$, or a post-main sequence $\mathrm{G}$ giant at $d \approx 300 \mathrm{pc}$ is still not clear. As a first-ascent giant, it is the first to be observed with a molecular and dust disc. Alternatively, BP Psc would be among the nearest $\mathrm{T}$ Tauri stars with a protoplanetary disc (PPD). We investigate whether the disc geometry resembles typical PPDs, by comparing polarimetric images with radiative transfer models. Our Very Large Telescope/Spectro-Polarimetric High-contrast Exoplanet REsearch (SPHERE)/Zurich IMaging Polarimeter (ZIMPOL) observations allow us to perform polarimetric differential imaging, reference star differential imaging, and Richardson-Lucy deconvolution. We present the first visible light polarization and intensity images of the disc of BP Psc. Our deconvolution confirms the disc shape as detected before, mainly showing the southern side of the disc. In polarized intensity the disc is imaged at larger detail and also shows the northern side, giving it the typical shape of high-inclination flared discs. We explain the observed disc features by retrieving the large-scale geometry with MCMAX radiative transfer modelling, which yields a strongly flared model, atypical for discs of T Tauri stars.
\end{abstract}

Key words: polarization - techniques: high angular resolution-techniques: polarimetricprotoplanetary discs - circumstellar matter-stars: evolution.

\section{INTRODUCTION}

Circumstellar discs appear at different stages of stellar evolution. During early stages of star formation, collapsing molecular clouds result in protoplanetary discs (PPDs) and later debris discs (Williams \& Cieza 2011). Although mass ejections of post-mainsequence stars are often spherical, they are known to create disclike surroundings for (post-)asymptotic giant branch (AGB) stars (Skinner, Meixner \& Bobrowsky 1998; Jeffers et al. 2014; Kervella et al. 2015).

Zuckerman et al. (2008, hereafter Z08) detect a circumstellar gas disc around BP Piscium (hereafter BP Psc) in ${ }^{12} \mathrm{CO}(3-2)$ with the Submillimeter Array (SMA) and in ${ }^{12} \mathrm{CO}(2-1)$ with the Owens Valley Radio Observatory interferometric measurements, and by

\footnotetext{
* The observations were taken during SPHERE science verification by the European Southern Observatory, Chile (ESO program ID: 60.A-9375(A)). †E-mail: deboer@strw.leidenuniv.nl
}

deconvolving Keck $H$ - and $K^{\prime}$-band images they detected a dust disc at high inclination $\left(i=75 \pm 10^{\circ}\right.$, with $i=0^{\circ}$ for a face-on disc) and position angle $\mathrm{PA}=118 \pm 5^{\circ}$. Since no reliable parallax has been determined, the distance $(d)$ to the star is highly uncertain. For two possible evolutionary scenarios with an effective temperature $T_{\text {eff }} \sim 5000 \mathrm{~K}$, the luminosities are matched to observations by varying $d$. Z08 propose two possible evolutionary scenarios for BP Psc: (1) at a distance $d \approx 80 \mathrm{pc}$, it is one of the nearest pre-main-sequence Classical T Tauri Stars (CTTSs) with an age $\approx 10 \mathrm{Myr}$; or (2) at $d \approx 300 \mathrm{pc}$, BP Psc is a post-main-sequence star of a few Gyr at its first-ascent, or hydrogen shell burning phase. A more luminous (therefore $d \sim 5000 \mathrm{pc}$ ) (post-) AGB (helium shell burning) star is ruled out due to its large Tycho 2 proper motion $\left(\triangle \mathrm{RA}=44.4 \pm 4.1 \mathrm{mas} \mathrm{yr}^{-1}, \Delta \mathrm{Dec} .=-26.3 \pm 4.3 \mathrm{mas} \mathrm{yr}^{-1}\right.$, $\mathrm{Høg}$ et al. 2000). For a star on the first-ascent giant branch, the associated molecular disc, accretion and Herbig Haro objects would be the first ever to be detected. The growing primary star would have recently enveloped a previous companion, hence creating the disc. Z08 favour the G giant scenario for BP Psc, mainly because of low 
lithium abundance, low surface gravity, and lack of an associated star-forming region. The stellar photosphere, obscured by the disc in visible and near-infrared (IR) wavelengths, is directly detected in X-rays with Chandra (Kastner et al. 2010). The authors argue that the ratio of X-ray over bolometric luminosity is too low for typical CTTSs but does agree with other rapidly rotating $\mathrm{G}$ giant stars. Furthermore, the $\mathrm{G}$ giant scenario is supported by the disc modelling of Melis et al. (2010, hereafter M10): the disc model for the Herbig Be star HD 100546 matches the spectral energy distribution (SED) of BP Psc, yielding an inner disc morphology which suggests that BP Psc is too luminous to be CTTS and leads them to suggest the presence of a massive planet carving a gap in the disc. However, early studies of PPDs (e.g. Andrews et al. 2011; Espaillat et al. 2011; Dong et al. 2012) have shown disc models based on the SED to be highly degenerate for many disc parameters, which can be solved by including high spatial resolution images of the discs in the analysis.

We compare visible light polarimetric images of BP Psc's disc with radiative transfer modelling to constrain the 3D geometry of the system. The recent increase of detected and modelled PPDs will allow a qualitative assessment of the CTTS scenario.

\section{OBSERVATIONS AND DATA REDUCTION}

\subsection{Observations with VLT/SPHERE/ZIMPOL}

We observed BP Psc and reference star TYC 5259-446-1 (T52) on 2014 December 6, during science verification of the SpectroPolarimetric High-contrast Exoplanet REsearch (SPHERE) instrument (Beuzit et al. 2008), the new high-contrast imager of the Very Large Telescope (VLT). We used the Zurich IMaging Polarimeter (ZIMPOL; Thalmann et al. 2008) in field tracking, polarimetric (P2) mode. We used the $R^{\prime}\left(\lambda_{0}=626.3 \mathrm{~nm} ; \Delta \lambda=148.6 \mathrm{~nm}\right)$ and $I^{\prime}\left(\lambda_{0}=789.7 \mathrm{~nm} ; \Delta \lambda=152.7 \mathrm{~nm}\right)$ filters simultaneously. The filter choice implied the use of the 'grey' beam splitter, which sends 20 percent of all visible light to the SPHERE eXtreme Adaptive Optics (Fusco et al. 2014) wave front sensor (WFS) and the remaining 80 per cent to ZIMPOL. The stellar magnitudes and air mass $a$ during observation of BP Psc ( $V=11.9 \mathrm{mag}, a=1.45 \pm 0.13$ ) and T52 $(V=11.4 \mathrm{mag}, a=1.14 \pm 0.02)$ posed an additional challenge for the WFS. As a result, we reached a Strehl ratio in the $R^{\prime}$ band of $S_{R^{\prime}} \sim 2.8$ per cent and a full width at half-maximum $\left(\mathrm{FWHM}_{R^{\prime}}\right)=50$ mas. In the $I^{\prime}$ band, we reached $S_{I^{\prime}} \sim 6.8$ per cent, $\mathrm{FWHM}_{I^{\prime}}=40$ mas.

We briefly summarize how ZIMPOL works, but refer to Schmid et al. (2012) for details on the instrument. A full polarimetric cycle consists of four exposures of $60 \mathrm{~s}$ for each of the four half wave plate (HWP) angles: $\theta_{\mathrm{hwp}}=0^{\circ}, 45^{\circ}, 22.5$, and 67.5. We recorded one polarimetric cycle (=16 min on target) for T52 and three polarimetric cycles (=48 min on target) for BP Psc. During each individual exposure, the Ferro-electric Liquid Crystal (FLC) reverses the polarization state, by switching its fast axis from the initial $(A)$ state at $0^{\circ}-45^{\circ}(B$ state $)$, at a frequency of $26.97 \mathrm{~Hz}(=1618 \times$ per integration). In the first frame (the 0 phase), 'charge shuffling' ensures that the light observed during the FLC's $A$ state is stored in the odd rows of the detector while the light observed in the $B$ state (where the measured polarization has changed sign) is stored in the even detector rows. For the next frame (the $\pi$ phase), the storage order is reversed: $A$ is saved in the even rows, $B$ in the odd rows. Therefore, the four exposures per $\theta_{\text {hwp }}$ consist of two ' $O$ frames' and two ' $\pi$ frames'.

\subsection{Polarimetric differential imaging}

We applied different filters to the two beams of the beam splitter. Therefore, we perform the reduction for each beam separately, with our custom-made reduction pipeline. After dark subtraction and flat-field correction, odd and even pixel rows are extracted into FLC $A$ and $B$ frames, which we subtract for the $O$ frames $\left(A_{0}-B_{0}\right)$ and the $\pi$ frames $\left(A_{\pi}-B_{\pi}\right)$. Next, we subtract the $\pi$ difference images from the 0 ones, and stack the two resulting images for each subsequent $\theta_{\text {hwp }}$. For $\theta_{\text {hwp }}=0^{\circ}$, we obtain the intensity $\left(I_{Q^{+}}\right)$and linear polarization $\left(Q^{+}\right)$by combining 0 and $\pi$ images:

$I_{Q^{+}}=0.5 \times\left.\left(\left(A_{0}+B_{0}\right)+\left(B_{\pi}+A_{\pi}\right)\right)\right|_{\theta_{\mathrm{hwp}}=0}$,
$Q^{+}=0.5 \times\left.\left(\left(A_{0}-B_{0}\right)-\left(B_{\pi}-A_{\pi}\right)\right)\right|_{\theta_{\mathrm{hwp}}=0}$.

Similarly, for $\theta_{\mathrm{hwp}}=45^{\circ}$, we obtain $I_{Q^{-}}$and $Q^{-} ; \theta_{\mathrm{hwp}}=22.5$ gives $I_{U^{+}}$and $U^{+}$; and $\theta_{\text {hwp }}=67.5$ yields $I_{U^{-}}$and $U^{-}$. To correct for charge trapping (a problem inherent to charge shuffling, described by Povel, Keller \& Yadigaroglu 1994 and Schmid et al. 2012), the aforementioned procedures are done before any centering routine is applied.

The initial separation of the odd and even pixel rows leads to different plate scales for the vertical and horizontal axes. We obtain 'square' $15 \times 15$ mas pixels by binning $4 \times 2$ pixels. We centre the images by cross-correlating the $I_{\theta_{\mathrm{hwp}}}$ images with a centred Moffat function and apply the same shift to the corresponding $Q^{+/-}$or $U^{+/-}$image. The final Stokes components are

$I_{Q}=0.5 \times\left(I_{Q^{+}}+I_{Q^{-}}\right)$,

$Q=0.5 \times\left(Q^{+}-Q^{-}\right)$,

$I_{U}=0.5 \times\left(I_{U^{+}}+I_{U^{-}}\right)$,

$U=0.5 \times\left(U^{+}-U^{-}\right)$

from which we can compute the polarized intensity $\mathrm{PI}=\left(Q^{2}+U^{2}\right)^{1 / 2}$, and the polarization angle $P_{\theta}=\arctan (U / Q)$.

\subsubsection{Correcting instrumental polarization}

Computing the difference $Q^{+}-Q^{-}$images (same for $U^{+/-}$) with equations (4) and (6) corrects for instrumental polarization (IP) created downstream from the HWP (Canovas et al. 2011, C11; Witzel et al. 2011; de Boer et al. 2014). However, this does not remove IP induced by the third mirror (M3) of the telescope and the first mirror (M4) of SPHERE, both of which are upstream from the HWP. We cannot distinguish between this instrumental and real (inter)stellar and/or disc polarization at the location of the star. C11 describe the correction for IP in imaging polarimetry, which assumes that the central star is unpolarized. Therefore, we consider any signal measured over a small aperture at the centre to be IP, which is the best we can do. Fig. 1 shows the IP-corrected PI images for $R^{\prime}$ band (left-hand panel), $I^{\prime}$ band (middle panel) and $R^{\prime}+I^{\prime}$ combined (right-hand panel). The purple lines in the $R^{\prime}$ - and $I^{\prime}$-band images show the direction of $P_{\theta}$.

\subsection{Reference star differential imaging (RDI)}

When we compare the Stokes $I$ [or total intensity $\left.I_{\mathrm{t}}=0.5 \times\left(I_{Q}+I_{U}\right)\right]$ images of BP Psc and the point source T52 in the two top panels of Fig. 2, we already see that BP Psc is not just 


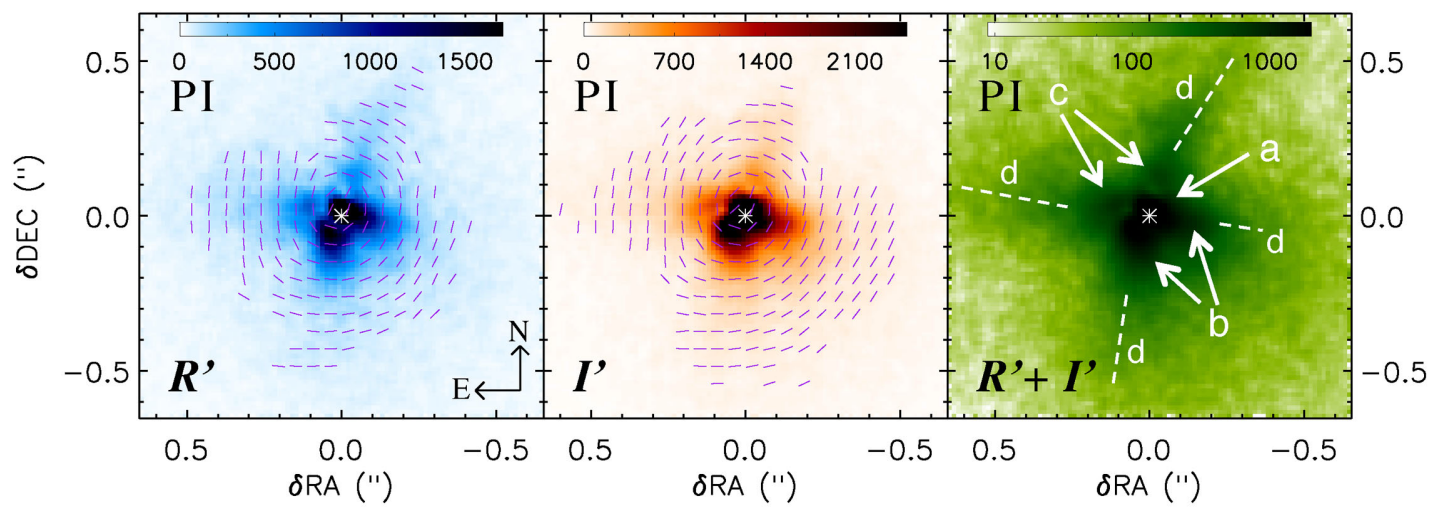

Figure 1. Polarized intensity (PI) image of BP Psc in $R^{\prime}$ band (left-hand panel); in $I^{\prime}$ band (middle panel); and both filters combined (right-hand panel). Where the signal is $>10$ times the background noise, purple lines show the polarization angle $P_{\theta}$. Note that the units are arbitrary and $R^{\prime}+I^{\prime}$ is presented in log scale. The three panels all show the same features: (a) a bright blob at position of the star centre (highlighted with a white asterisk); (b and c) are the southern and northern 'bowls' of the highly inclined disc; (d) extended fingers, aligned with both the brighter bowls of the northern and southern disc.

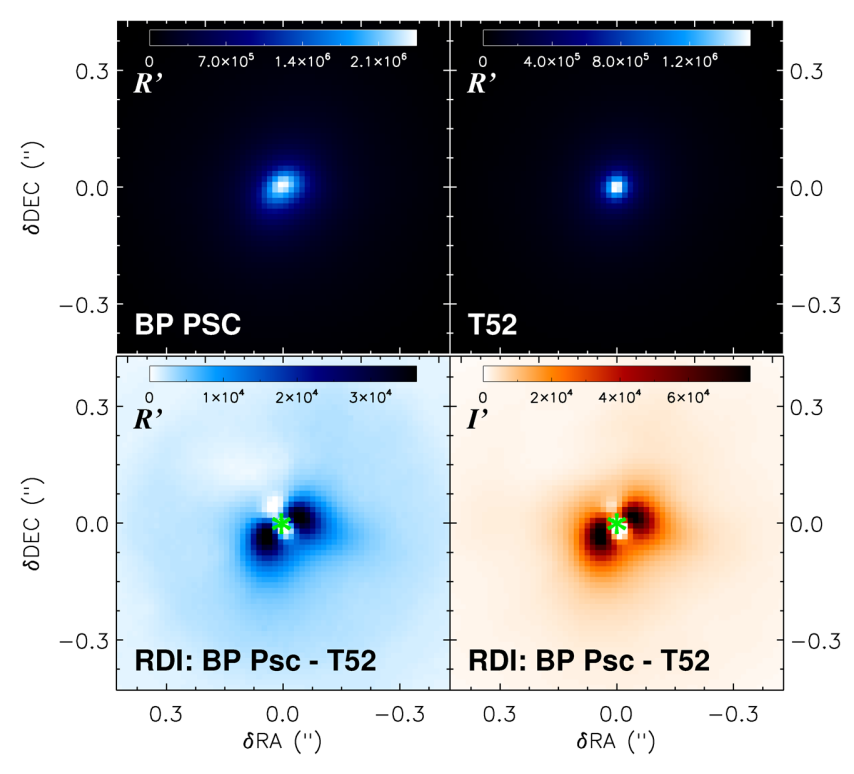

Figure 2. Top panel: $R^{\prime}$-band $I_{\mathrm{t}}$ image of BP Psc (left-hand panel) and T52 (right-hand panel). Bottom panel: RDI $\left(I_{\mathrm{t}, \mathrm{BP} P s c}-I_{\mathrm{t}, \mathrm{T} 52}\right)$ images of the disc in $R^{\prime}$ band (left-hand panel) and $I^{\prime}$ band (right-hand panel). The green asterisk shows the position of the star.

a point source: the disc contributes a significant part of the signal. T52 was observed at lower air mass than BP Psc (see Section 2.1), which results in a smaller FWHM and higher Strehl ratio for T52. For RDI (Smith \& Terrile 1984), where we subtract a reference star from our target, this difference in FWHMs becomes a problem, which could lead to oversubtraction (i.e. removal of disc light) close to the star and undersubtraction (insufficient removal of stellar speckle halo) at larger separations.

To adjust the point spread function (PSF) of T52 to the lower Strehl ratio of the BP Psc observations, we convolve T52 with a Gaussian of FWHM $=15$ mas to match the width of the $I_{\mathrm{t}}$ image of BP Psc at PA $=30^{\circ}$, which is roughly perpendicular to the disc PA of Z08. In this direction, we expect the influence of the disc on the shape of the PSF to be negligible. Finally, we scale the peak flux of the reference PSF to match the peak value for BP Psc and

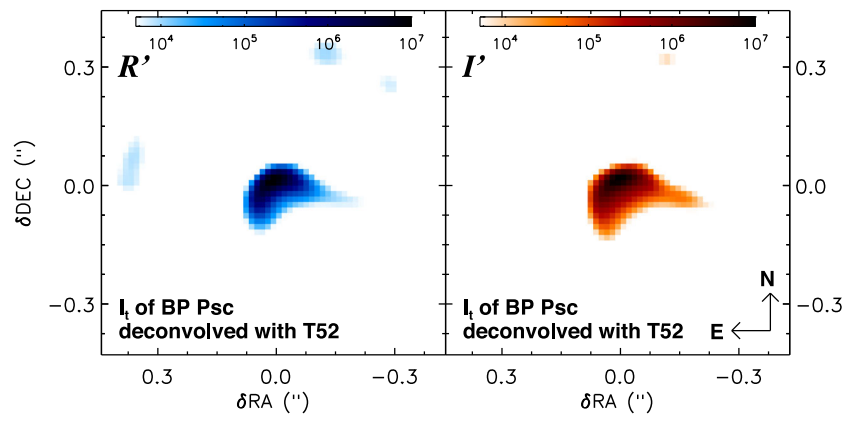

Figure 3. $I_{\mathrm{t}}$ image of BP Pscdeconvolved with the $I_{\mathrm{t}}$ image of T52 in $R^{\prime}$ band (left-hand panel) and $I^{\prime}$ band (right-hand panel). The deconvolution was performed with the RL method.

subtract the former from the latter. The bottom panels of Fig. 2 show the RDI images for $R^{\prime}$ (left-hand panel) and $I^{\prime}$ ( right-hand panel).

\subsection{Deconvolution of the total intensity image}

The only images of the resolved disc known to date are the deconvolved images of Z08, which display a different structure than our PI images in Fig. 1. To confirm the detection of Z08, we apply the same method and perform the Richardson-Lucy (RL) deconvolution (using equations 19 and 20 in Lucy 1974) of the $I_{\mathrm{t}}$ image of BP Psc, for which we show in Section 2.3 that the disc signal forms a substantial part. The observed $I_{\mathrm{t}}$ is used as a starting guess for the deconvolution. As in Section 2.3, the PSF is obtained by convolving the $I_{\mathrm{t}}$ image of T52 with a Gaussian $(\mathrm{FWHM}=15$ mas). RL recovers structures on scales larger than the FWHM within only a few $(\sim 100)$ iterations. Because our FWHM is comparable to the angular size of the disc, we require more iterations to converge. To monitor the convergence of the deconvolution, we convolve the deconvolved images and subtract this from the original $I_{\mathrm{t}}$ images. After 2000 iterations, the residuals show little change. Fig. 3 shows the final RL deconvolved images for both filters. 


\section{RESULTS}

We detect the disc of BP Psc for the first time in PI in both $R^{\prime}$ and $I^{\prime}$ bands (Fig. 1). Additionally, we retrieve the intensity image of the disc with RDI in Fig. 2, and confirm the detection of Z08 with the very similar results of our deconvolution (Fig. 3). The overall appearance of the PI images of Fig. 1 is the same for both filters, and resembles an (nearly) edge-on flaring disc, similar to e.g. HH 30 (Burrows et al. 1996, CTTS with $i>80^{\circ}$ ). To give a flux-calibrated surface brightness of the disc requires a detailed calibration of (e.g. the polarimetric efficiency of) ZIMPOL, which is beyond the scope of this Letter. We confine this study to a qualitative description and analysis of the observations. The main features are highlighted in the right-hand panel of Fig. 1. A bright blob appears in the centre of the images (feature a) covering the position of the star (highlighted with a white asterisk). The two bright regions [at position angles (PA $\sim 160^{\circ}$ and $\sim 270^{\circ}$ ) within 0.15 arcsec from the star (feature b) show the 'bowl' of a flared disc south of the mid-plane. This southern bowl dominates both the RDI images (Fig. 2) and the deconvolutions (Fig. 3) as well. For the northern counterpart of this bowl, only two elongated regions are detected in the PI images (features $\mathrm{c}$ in Fig. 1). However, two faint extended 'fingers' (feature d in Fig. 1), starting at the feature $\mathrm{c}$, going outward to $\sim 0.5 \operatorname{arcsec}$ seem to trace the extended surface of the flared disc, north of the mid-plane. Less clearly, similar fingers can be seen extending outward to the south and west from the $\mathrm{b}$ regions.

Compared to high Strehl ratios reached for much brighter, $R<8$ mag stars $\left(S_{R^{\prime}} \approx 60\right.$ per cent, Fusco et al. 2014), where high angular resolutions are reached (e.g. FWHM $R_{R^{\prime}} \lesssim 20$ mas for Kervella et al. 2015), the low Strehl ratios reached for BP Psc $\left(R \approx 11 \mathrm{mag}, S_{R^{\prime}} \approx 2.8\right.$ per cent) naturally result in lower resolution: $\mathrm{FWHM}_{R^{\prime}}=50$ mas. Both RDI and RL deconvolution are very sensitive to errors in the PSF. Due to the varying Strehl ratios, T52 can only be considered as an approximate PSF (e.g. we have to broaden T52 to fit the FWHM of BP Psc). Therefore, we do not put too much emphasis on either the deconvolutions or the RDI images, we only emphasize that our deconvolved images in Fig. 3 look very similar to the ones obtained by Z08 in $H$ and $K$ bands. We base the main focus of our analysis on the PI images in Fig. 1.

\section{DISCUSSION}

\subsection{Modelling the disc}

To find the evolutionary stage of BP Psc, ideally we would compare disc models to those typical for discs around CTTSs and those around $\mathrm{G}$ giants. To the best of our knowledge, no radiative transfer models exist for discs around other first-ascent giants. Models exist for more evolved systems (e.g. Jeffers et al. 2014; Kervella et al. 2015) However, a disc around a first-ascent giant must have formed very recently, which makes our knowledge on the largescale geometry of such systems very uncertain. Therefore, we do not consider a comparison of this system to AGB disc models as a viable option. Instead, we choose to treat BP Psc as if it were a CTTS, at $d=81 \mathrm{pc}$, represented by a Kurucz stellar model atmosphere (Kurucz 1979) with surface temperature $T=5000 \mathrm{~K}$, luminosity $L=0.67 \mathrm{~L}_{\odot}$, and mass $M=1.1 \mathrm{M}_{\odot}$, and see if our best model resembles those created for discs around other CTTSs. The disc radii and scaleheight $(H)$ do essentially scale with distance, while leaving the overall shape unchanged. We do not aim to describe the dust properties of the disc, rather constrain the disc geometry by creating a model which fits the SED, while producing images which capture the major features of our observations.

We use the Monte Carlo radiative transfer code MCMAX (Min et al. 2009) ${ }^{1}$ to fit the SED of BP Psc. The disc models are made up of silicates and contain carbon with a carbon mass fraction $M_{\text {carbon }} /\left(M_{\text {silicates }}+M_{\text {carbon }}\right)=0.1$, which is comparable to the fraction in the Solar system. Grain sizes lie between $0.05 \mu \mathrm{m}$ and $3 \mathrm{~mm}$ and decrease with a power law. Similar to the results of M10, our models contained two disc components: an inner disc at $0.12 \leq$ $r \leq 1$ au and an outer disc at $r \geq 1.5$ au. The scaleheight $(H)$ of both $(j=$ inner, outer) disc components increases with a power law $H(r) \propto r_{j}^{p}$ (equation 18 of M09). The inner disc maintains this power-law component $\left(p_{\text {in }}\right)$ for all radii. The increase of the outer disc goes with the power of ( $p_{\text {out }}$ ) up to a radius $r_{\exp }$ from where $H$ declines exponentially, causing a rapid decrease in the scattering of starlight. The scaleheight decreases with particle size, due to settling of large dust grains towards the disc mid-plane. Dust settling is higher (or $H_{\text {dust }} / H_{\text {gas }}$ is smaller) when turbulent mixing decreases. Turbulent mixing is described by the viscosity parameter $\alpha_{\text {turb }} \propto$ $\left(H_{\text {dust }} / H_{\text {gas }}\right)^{2}$ (Dubrulle, Morfill \& Sterzik 1995; Woitke et al. 2016).

We allowed the inclination $(i)$ to vary such that the line-of-sight optical depth at $550 \mathrm{~nm}$ is $\tau_{550}=5.2$, and found an inverse relationship between $H$ and $i$. Even though we created fits to the SED with similar quality with a variety of parameters, the ZIMPOL observations placed lower limits on $H$, and therefore on $p_{\text {out }}$, which in turn required an $i \lesssim 80^{\circ}$. A degeneracy exists between the disc dust mass ( $\left.M_{\text {dust }}\right)$ and $\alpha_{\text {turb }}$ for the fit of the near- and mid-IR range, where lower $M_{\text {dust }}$ require higher values of $\alpha_{\text {turb }}$ (i.e. settling becomes less efficient). The mass of the outer dust disc has a lower limit beneath which $i$ is no longer affected by $p_{\text {out }}$ and only near edge-on configurations could still maintain the $\tau_{550}$ requirement. This lower limit of $M_{\text {dust, out }} \gtrsim 10^{-7} \mathrm{M}_{\odot}$ effectively gives us an upper limit for the viscosity parameter $\alpha_{\text {turb }} \lesssim 5 \times 10^{-5}$. This means that the dust settling in the disc of BP Psc is much stronger than the example of strong settling given by Woitke et al. (2016), who use $\alpha_{\text {turb }}=10^{-4}$.

The final model (Fig. 4) has $i=78.9$ (in agreement with $i=75 \pm 10^{\circ}$ of Z08); $r_{\exp }=30$ au, $\alpha_{\text {turb }}=5.0 \times 10^{-6}$, and $p_{\text {in }}=1.14$ and $p_{\text {out }}=3.0$ for the inner and outer disc, respectively. The model fits the SED very well, except for a strong silicate feature at $10 \mu \mathrm{m}$. We have found that increasing the carbon/silicate fraction can remove this feature, but using this chemical composition we did not achieve a good fit to the SED overall so far. Since we consider the chemical composition of the disc to be outside of the scope of this Letter, we did not pursue the removal of the silicate feature any further. The Stokes images of the disc model in the direction of the line of sight are convolved with the $I_{\mathrm{t}}$ image of the reference star, after which we applied the same procedure of IP correction as we did for the observations. The model will not truly be plagued by such an artefact, but as we explain in Section 2.2.1, we cannot distinguish between true polarization at the location of the star and IP in our observations. Applying the IP correction therefore ensures the best comparison between model and observation (as demonstrated by Min et al. 2012).

Fig. 4 shows for $I^{\prime}$ band the unconvolved model $I_{\mathrm{t}}$ and PI images (top left-hand panel and right-hand panel, respectively), and the PI image after convolution with the PSF (bottom left-hand panel), and IP correction (bottom right-hand panel). The convolved + IP corrected image shows a striking similarity with the reduced PI

\footnotetext{
${ }^{1}$ See the MCMAX website: http://www.hetisikke.nl/mcmax/ or the manual https://sites.google.com/site/manualmcmax/home.
} 

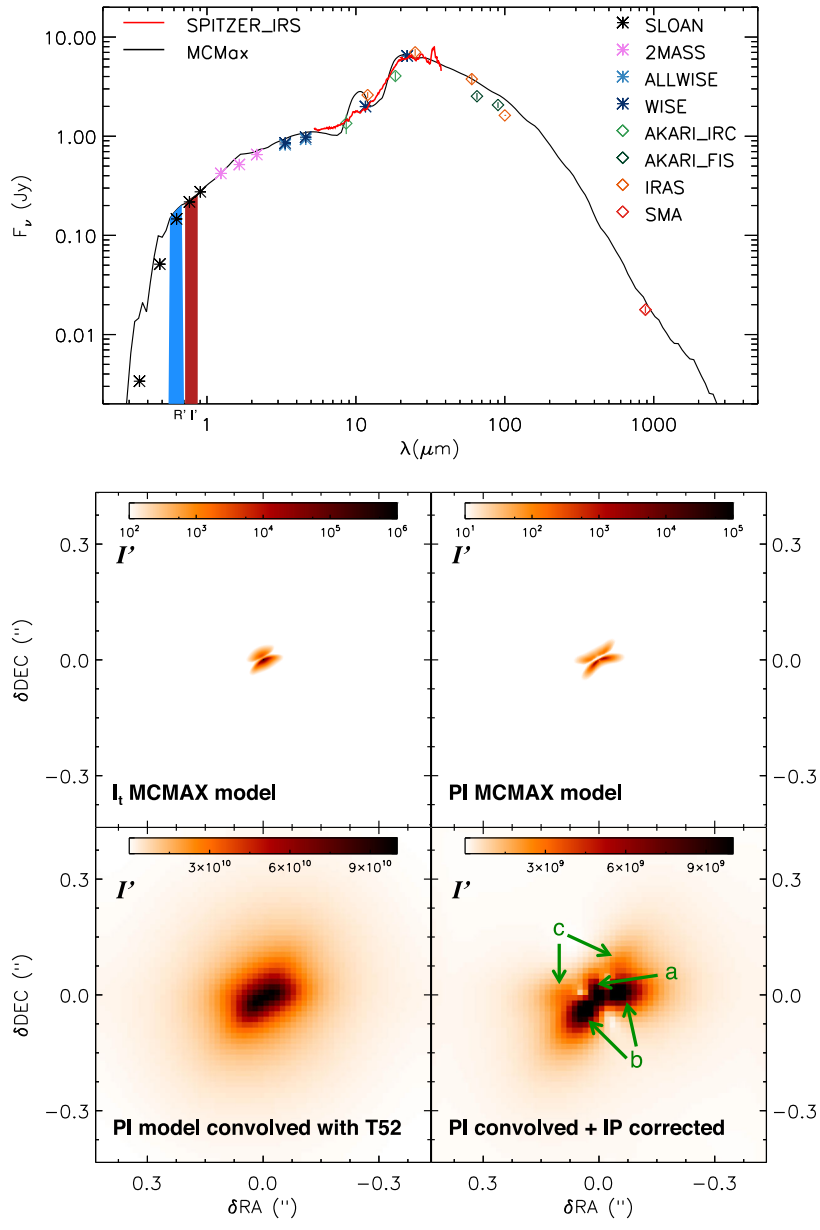

Figure 4. Top panel: SED of the MCMAX model along the line of sight at $i=78.9$ compared to the photometric measurements and IRS spectrum. The blue and red bars indicate the ZIMPOL $R^{\prime}$ and $I^{\prime}$ filter bands, respectively. The centre and bottom rows show the images corresponding to the MCMAX model. Centre in log scale: not accounted for telescope resolution and IP the $I_{\mathrm{t}}$ image (left-hand panel), and the PI image (right-hand panel). Bottom in linear scale: the same PI model image after convolution with the reference star (left-hand panel); the image on the right-hand panel is subsequently corrected for the signal at the centre, (as if it were IP) as described in Section 2.2.1. The features $\mathrm{a}, \mathrm{b}$, and $\mathrm{c}$ are comparable to the features with the same labels in Fig. 1.

images of Fig. 1. The features a, b, and c, described in Section 3, are visible in the convolved + IP corrected model image. Comparing the top-right and bottom-right images of Fig. 4 teaches us that features $\mathrm{b}$ and $\mathrm{c}$ represent the upper and lower arcs of a nearly edge-on disc, while feature a is an artefact, caused by the convolution + IP correction. We did not achieve to create a model with features, similar to d. However, from the morphology of the unresolved disc model, we can deduce that they are likely to be extensions of the northern arc of the disc. This explanation is supported by the fact that the $\mathrm{c}$ feature lie at the base of the $\mathrm{d}$ feature. In an alternative explanation, the second disc component is surrounded by a third and outermost disc component.

The angle between the northern and southern arc $(\sim 2 H / r)$ seems to be smaller for the model than for the ZIMPOL observations. Therefore, we do not claim to have found a unique solution for either $i$ or $H$ of the disc. We rather created a model with a morphology comparable enough to explain the observations. Our model is strongly flared for its small grains, but extremely flat for the larger grains. ALMA long baseline observations should be able to confirm if our modelling results are correct. Since small dust grains are coupled to the gas, we predict that the molecular disc shows a much stronger flaring than the large grain dust disc.

The parameters (especially flaring and settling) used for the presented model are far from the classical Keplerian disc in hydrostatical equilibrium, which means that the disc of BP Psc is not a stereo-typical disc for a CTTS. This could be explained by a very young star, with infall from its native star-forming nebula. However, since no associated star-forming region has been found, we do not consider this to be a plausible scenario. An alternative explanation for the atypical behaviour of the disc is that it is not a PPD surrounding a pre-main-sequence star, but rather a disc around a first-ascent G giant. Unfortunately, we lack G-giant disc models which allow for a proper comparison. An open question remaining for the G-Giant scenario is whether the formation of the disc in a way as proposed by Z08 and M10 (i.e. enveloping a massive companion) is compatible with the strong flaring of small grains and settling of the larger grains.

\subsection{Disc morphology}

Even though both sides of a strongly inclined (but $i \neq 90^{\circ}$ ) disc will be dominated by forward scattering, we expect the forward facing side (or top) to be brighter than the backward facing side (or bottom). The asymmetry originates from the smaller optical depth of the forward facing side of the disc (the starlight reaches us more efficiently). From the MCMAX model images, we determine that the top side of the disc is pointing south and the bottom side north. An interesting test to confirm that the southern side is facing us will be to check whether the associated southern HH-object (Z08) is blue shifted, and the northern counterpart red shifted.

We determine the PA by assuming (as we see for the disc model images in Fig. 4) that the largest symmetry will be across the axis $\mathrm{PA} \pm 90$, while the symmetry across the PA will always be broken for a disc with $i \neq 90^{\circ}$. We mirror the disc image along the PA and subtract the mirror image from the original disc image. For regions where the SNR is high in the original image, we take the absolute value of the residual (image mirror). We repeat this method for varying PA. The angle which provides the smallest residual signal yields $\mathrm{PA}=120.8 \pm 2.0$, in good agreement with $\mathrm{Z08}\left(\mathrm{PA}=118 \pm 5^{\circ}\right)$.

\section{CONCLUSION}

Our ZIMPOL observations of BP Psc confirm the presence of a circumstellar disc. Despite a modest AO correction, we resolved the disc for the first time in the visible ( $R^{\prime}$ and $I^{\prime}$ bands), and present the first polarimetric images of this object. Our deconvolved image in Fig. 3 confirms the disc images of Z08, and retrieve the PA with a higher accuracy than was known until now.

The MCMAX modelling yields images comparable to the observations. They require a model which is strongly flared for small grains, yet strong settling occurs for large grains. Both flaring and settling values are atypical for a PPD of a T-Tauri type star. Without strongly discarding the CTTS scenario, our study therefore is more inclined towards a G-giant evolutionary stage for this system. The comparison between model and observations allows us to determine that the forward facing side is pointing south $\left(\approx 211^{\circ}\right)$, while the backwardfacing side points north $\left(\approx 31^{\circ}\right)$. The specific disc features detected in Fig. 1 can be explained by the model as either resolved components of the forward-facing side of the disc (' $b$ ' features); the 
backward-facing side ('c' and 'd' features) or as a residual of the convolution with the telescope PSF and IP subtraction ('a' feature).

\section{ACKNOWLEDGEMENTS}

We are thankful to the European Southern Observatory (ESO) support staff on Paranal and the SPHERE science verification team for their support and the successful observations. HC acknowledges support from the Spanish Economía, Industria y Competitividad grant AYA 2014-55840-P.

\section{REFERENCES}

Andrews S. M., Wilner D. J., Espaillat C., Hughes A. M., Dullemond C. P., McClure M. K., Qi C., Brown J. M., 2011, ApJ, 732, 42

Beuzit J.-L. et al., 2008, in McLean I. S., Casali M. M., eds, Proc. SPIE Conf. Ser. Vol. 7014, Ground-based and Airborne Instrumentation for Astronomy II. SPIE, Bellingham, p. 701418

Burrows C. J. et al., 1996, ApJ, 473, 437

Canovas H., Rodenhuis M., Jeffers S. V., Min M., Keller C. U., 2011, A\&A, 531, A102 (C11)

de Boer J., Girard J. H., Mawet D., Snik F., Keller C. U., Milli J., 2014, in Ramsay S. K., McLean I. S., Takami H., eds, Proc. SPIE Conf. Ser. Vol. 9147, Ground-based and Airborne Instrumentation for Astronomy V. SPIE, Bellingham, p. 87

Dong R. et al., 2012, ApJ, 750, 161

Dubrulle B., Morfill G., Sterzik M., 1995, Icarus, 114, 237

Espaillat C., Furlan E., D’ Alessio P., Sargent B., Nagel E., Calvet N., Watson D. M., Muzerolle J., 2011, ApJ, 728, 49
Fusco T. et al., 2014, in Marchetti E., Close L. M., Véran J.-P., eds, Proc. SPIE Conf. Ser. Vol. 9148, SPIE Astronomical Telescopes + Instrumentation. SPIE, Bellingham, p. $91481 \mathrm{U}$

Høg E. et al., 2000, A\&A, 355, L27

Jeffers S. V. et al., 2014, A\&A, 572, A3

Kastner J. H., Montez R., Jr, Rodriguez D., Grosso N., Zuckerman B., Perrin M. D., Forveille T., Graham J. R., 2010, ApJ, 719, L65

Kervella P. et al., 2015, A\&A, 578, A77

Kurucz R. L., 1979, ApJS, 40, 1

Lucy L. B., 1974, AJ, 79, 745

Melis C., Gielen C., Chen C. H., Rhee J. H., Song I., Zuckerman B., 2010, ApJ, 724, 470 (M10)

Min M., Dullemond C. P., Dominik C., de Koter A., Hovenier J. W., 2009, A\&A, 497, 155

Min M., Canovas H., Mulders G. D., Keller C. U., 2012, A\&A, 537, A75

Povel H. P., Keller C. U., Yadigaroglu I. A., 1994, Appl. Opt., 33, 4254

Schmid H.-M. et al., 2012, Proc. SPIE Conf. Ser. Vol. 8446, Ground-based and Airborne Instrumentation for Astronomy IV. SPIE, Bellingham, p. 84468

Skinner C. J., Meixner M., Bobrowsky M., 1998, MNRAS, 300, L29

Smith B. A., Terrile R. J., 1984, Science, 226, 1421

Thalmann C. et al., 2008, in McLean I. S., Casali M. M., eds, Proc. SPIE Conf. Ser. Vol. 7014, Ground-based and Airborne Instrumentation for Astronomy II. SPIE, Bellingham, p. 70143F

Williams J. P., Cieza L. A., 2011, ARA\&A, 49, 67

Witzel G. et al., 2011, A\&A, 525, A130

Woitke P. et al., 2016, A\&A, 586, A103

Zuckerman B. et al., 2008, ApJ, 683, 1085 (Z08)

This paper has been typeset from a $\mathrm{T}_{\mathrm{E}} \mathrm{X} / \mathrm{L} \mathrm{T} \mathrm{E} X$ file prepared by the author. 